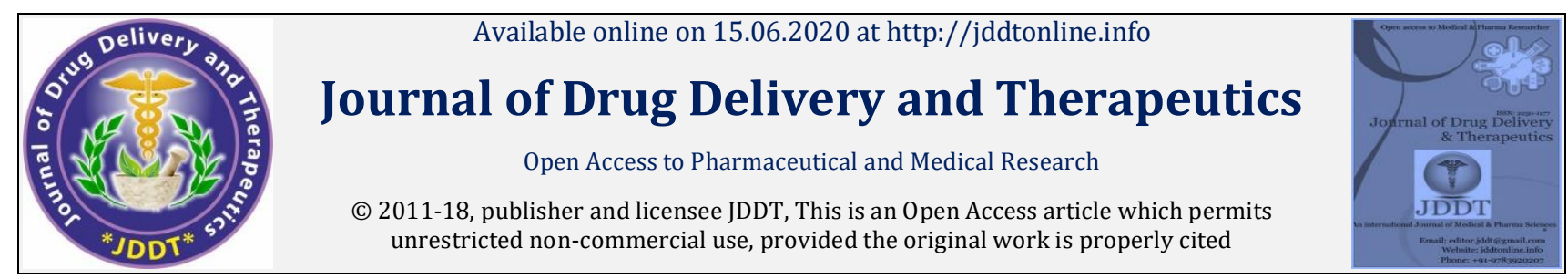

Open $\odot$ Access

Review Article

\title{
A Review: Divergent Emerging Strategies to Tackle Challenging Covid-19
}

\section{Puri Sudha U., Bhimanwar Arti A., Vaidya Mrunal M.}

JSPM's Jayawantrao Sawant College of Pharmacy and Research, Hadapsar, Pune-28, India

\begin{abstract}
Background: Contemplating the severity of pandemic sequel for public health many researchers are working recklessly for new targeted drug s and vaccines. Despite the challenging opportunities, the setup for the development of new effective remedy against COVID-19 is unforeseeable.

Objective: The aim of the present review was to explore the work done by some authors to battle against COVID-19.

Material \& Method: We analysed 24 articles from Elsevier and one from J Med virology journals stating different strategies to cope with this recently arrived monster COVID-19. Further are some targeting therapies viz., Niclosamide, immune response generated by cytotoxic T-cells and Natural Killer cells, Chloroquine and Hydroxy Chloroquine, blockade of Interleukin17, N- Acetylcysteine, mucolytic agents, oxytocin, bioflavonoids, triterpenoid saponins, ACE inhibitors or AII receptor antagonists, Hyperbaric oxygen therapy, serum albumin, Ritonavir, Tocilizumab, Furin, Amantadine, pioglitazone, Dapsone and doxycycline, steroids, plasama therapy, routine childhood immunization, a datadriven hypothesis on the epigenetic dysregulation of host metabolism by SARS corona viral infection, lipid metabolism has been proposed as a treatment target for COVID-19, COVIDiagnosis-Net proposed network for quick and accurate diagnosis.
\end{abstract}

Result: We got to know how the people are struggling to find a cure for this pandemic affair.

Conclusion: Till date, there is no specific treatment that has been confirmed to the effect against COVID-19. Let's hope for the best.

Key words: COVID-19, Novel Corona Virus Infection-2019, SARS-CoV2, Plasma therapy, Immunomodulators, etc.

Article Info: Received 22 March 2020; Review Completed 14 May 2020; Accepted 20 May 2020; Available online 15 June 2020

Cite this article as:

Puri SU, Bhimanwar AA., Vaidya MM, A Review: Divergent Emerging Strategies to Tackle Challenging Covid-19, Journal of Drug Delivery and Therapeutics. 2020; 10(3-s):251-255 http://dx.doi.org/10.22270/jddt.v10i3-s.4117

*Address for Correspondence:

Prof. Puri Sudha U. M. Pharm, JSPM's Jayawantrao Sawant College of Pharmacy and Research

\section{INTRODUCTION:}

The outbreak of novel coronavirus disease 2019 (COVID-19) which was originated in Wuhan, Hubei, China, in December 2019, has been declared as public health emergency of international concern by World Health Organization (WHO). On 30 January 2020, the WHO Director-General declared that the current outbreak constituted a public health emergency of international concern, and on 12 March 2020 the COVID-19 outbreak was declared a pandemic [1]. In the face of rapidly spreading disease and a large number of infected people, there is an urgent need for effective COVID19 prevention and control measures. WHO has declare that there are currently no known treatment for COVID-19 and does not recommend the use of antiviral drugs, antibiotics, glucocorticoids, or traditional Chinese medicine. Even that, there have been reports of the use of many antibiotics and steroids viz., oseltamivir, lopinavir /ritonavir, prednisone, antibiotics, and traditional Chinese medicine for the treatment of patients with COVID-19 [2].
Contemplating the severity of pandemic sequel for public health many researchers are working recklessly for new targeted drugs and vaccines. Despite the challenging opportunities, the setup for the development of new effective remedy against COVID-19 is unforeseeable.

In this present review we focused on different emerging strategies to gear up with SARS-CoV-2. Here are some proposed hypotheses for the treatment of COVID-19 by some authors from reputed journals using diverse types of targeted therapies to brawl against it.

\section{SOME PROPOSED HYPOTHESES:}

Drug repurposing is an attractive approach for bringing new drugs quickly into market. Various drug repurposing screens identified NIC as a potential drug candidate against COVID19. Prevention of viral entry by altering endosomal $\mathrm{pH}$ and prevention of viral replication by inhibition of autophagy are the plausible mechanisms of NIC against COVID-19. SARSCoV-2 infects the permissive cells through the receptor of angiotensin converting enzyme -2 (ACE-2). Spike (S) 
protein, 3C-like main protease, NTPase / helicase, RNA dependent RNA polymerase (RNA replicase) and host receptors are the crucial drug targets of SARS-CoV-2. NIC was reported to $\mathrm{pH}$ dependent endocytosis of human rhino virus (HRV) and influenza. This mechanism may also contribute to the antiviral effects of NIC against SAR-CoV-2. Moreover, NIC can prevent autophagy of SARS-CoV-2 by inhibition of S-Phase kinase associated protein 2 (SKP2). Clinical efficacy of NIC against COVID-19, therefore, need to be evaluated against COVID-19.

S.K.S.S. Pindiprolu and S.H. Pindiprolu hypothesized, the potential antiviral mechanisms of (Niclosamide) NIC against COVID-19. They utilized an attractive approach that is, "Drug repurposing, by employing 'old' drugs to treat 'new' diseases in drug discovery" by using Niclosamide (NIC) which is an approved anti-helminthic drug with diverse antiviral mechanisms [3].

A. Yaqinuddin and J. Kashir suggested that the Cytotoxic Tcells (CTLs) and Natural Killer (NK) cells are essential to generate an effective immune response against resultant disease (COVID-19) which is more severe in individuals with a weak immune system and patients with severe COVID-19 which present significantly lower lymphocyte, and higher neutrophil, counts in blood specifically, CD8+ lymphocytes and NK cells compared to patients with mild infection and healthy individuals. COVID-19 severity could be treated via Interferon therapy to generate adequate immune response, use of chloroquine, broad-based antibiotics and antivirals to limit viral replication and co-infections and use of Monalizumab to restore the function of CD8+ T and NK cells. It is observed that the patients with COVID-19 showed significantly lower lymphocyte and higher neutrophil counts in blood compared to healthy controls. The NK group 2 member A (NKG2A) heterodimeric receptor is one of the most prominent NK cell inhibitory receptors ${ }^{[4]}$.

Over-expression of NKG2A (an inhibitory receptor) on CD8+ and NK cells of COVID-19 infected patients compared to healthy controls has been demonstrated recently. Suppression of the innate immune response occurs via overexpression of NKG2A (an inhibitory receptor) on CD8+ and NK cells, leading to their reduction and an increase in opportunistic and coinfections of the lung in COVID-19 patients with severe symptoms [4].

Chloroquine (CQ) and Hydroxy Chloroquine (HCQ) with immunomodulatory and anti-inflammatory effects, enter the cytoplasmic vesicles through the cytoplasmic membrane, and increase the $\mathrm{pH}$ from 4.0 to 6.0 , and due to reduced $\mathrm{pH}$ acid-dependent sub cellular functions get inhibited, antigen processing in macrophages will be impaired and receptor binding and membrane fusion will also get inhibited. Moreover, the replication of the virus will block due to the change in $\mathrm{pH}$ required for lysosome and enzyme activities. Due to the mechanisms of action of these drugs, over activation of the immune system triggered by SARS-CoV-2 may be suppressed and the progression to severe disease will be controlled [5].

It is observed that IL-17 increased in intensive-care COVID19 patients vs non intensive-care and controls hypothesizing that blocking IL-17 could have the potential to improve COVID-19s aberrant immune response and acute respiratory distress syndrome-related mortality. A cytokine that may be related to IL- 6 in the condition of viral infection is IL-17. Hou et al. found in murine viral models that the superfluous IL-6 level promotes the generation of Th17 cells, and the resulting IL-6 and IL-17 synergistically promote viral obstinacy by shielding virus infected cells from apoptosis. IL17 blocking role in COVID19 subjects with cytokine storm risk as the COVID-19 patients showed lower leukocyte numbers, abnormal respiratory findings, and increased levels of plasma pro-inflammatory cytokines such as interleukin (IL)1- $\beta$, IL1RA, IL6, IL8, IL10 with Th1-Th17 cells appearing as the main source which appears as a potential promising therapeutic target [6].

5-alpha-reductase inhibitors may disrupt androgens metabolism in lungs, which in turn may control COVID-19 infection. Finasteride and dutasteride both of these members of 5-alpha-reductase inhibitors are used to reduce prostate volume in Benign Prostatic Hyperplasia by blocking 5 -alpha-reductase isoform 3 , which is expressed in respiratory epithelium and fibroblasts. It is achieved that androgens including DHT disrupt broadcasting between fibroblasts and alveolar type II cells by a mechanism involving TGF $\beta$ (Transforming Growth Factor beta) and EGF (Epidermal Growth Factor) receptor signaling pathways. 5alphareductase inhibitors might enhance androgen concentration in lungs impeding their regeneration. Interstitial pneumonia is the main cause of life-threatening respiratory disorders at the severe stage of COVID-19 infection [7].

$\mathrm{N}$-Acetylcysteine (NAC), a well-known mucolytic agent used in respiratory infections, is a thiol-containing free-radical scavenger and a precursor of glutathione. The beneficial action of $1200 \mathrm{mg} / \mathrm{d}$ of oral NAC in respiratory diseases has been previously demonstrated in prevention of chronic obstructive pulmonary disease exacerbations and addition of this dose of NAC to conventional treatment improves oxidative stress and inflammatory response also. In the viral lower respiratory tract infections associated with of IL-8, IL6 , and TNF- $\alpha$ expression and release in alveolar type II cells infected with influenza virus $\mathrm{A}$ and $\mathrm{B}$ and respiratory syncytial virus is inhibited by NAC. Thus, at the dose 1200 $\mathrm{mg} / \mathrm{d}$ oral NAC used in patients with COVID- 19 , as a measure that could potentially prevent the development of the cytokine storm syndrome and ARDS [8].

Oxytocin (OT) a nonapeptide hormone acting in the body and the brain, constitutes a biological target against coronavirus infection, especially in high-risk population, with underlying conditions including diabetes, high blood pressure, cardiovascular issues and obesity. In humans, activation of the OT receptor, which are expressed by endothelial cells in the pulmonary artery, produce a vasodilatory effect via stimulation of the nitric oxide (NO) pathway which is a key signaling molecule acting as a host response modulator in viral infections [9].

Bioflavonoids are well known for many biological and therapeutic effects including antioxidant and antiviral properties. Protease MPro and the papain-like protease are prime importance for processing polyproteins translated from the viral RNA. Recent studies showed the human monoclonal 47D11 antibody exerted its activity to neutralize SARS-CoV-2 binding a conserved epitope on the spike receptor binding domain. This receptor action is performed through a mechanism independent from the receptor binding inhibition and could be useful to prevent or treat SARS-CoV-2 infection [10].

Saikosaponin A, Saikosaponin B, and Saikosaponin D are major triterpenoid saponins derived from Bupleurum falcatum L. (Umbelliferae) with valuable pharmacological activities. These active components exbibit several immunomodulatory, anti-inflammatory, anti-bacterial, antiviral, and anti-cancer effects. Saikosaponin A dosedependently inhibits the production of several inflammatory mediator's ROS, TNF-a, COX-2, iNOS, and interleukins (IL-6, IL-8, and IL-10) which are responsible for the cytokine 
storm of severe COVID-19 patients. Saikosaponin D could exhibit an anti-proliferative effect in activated T-lymphocyte, in part via suppression of the nucleotide-binding oligomerization. Saikosaponin A inhibited influenza A virus and reduced lung immunopathology. Saikosaponin B demonstrated in-vitro antiviral activity against HCoV-229E by inhibiting the viral attachment to cells in a dosedependent manner, blocking the viral penetration into cells, and interfering with the early stage of viral replication, such as virus absorption and penetration. Saikosaponin D inhibits the autophagic activity of Enterovirus A71. All suggest that Saikosaponins might hold the promise for the treatment of COVID-19 [11].

Renin-angiotensin system is the hormone system which regulates blood pressure and vascular resistances, as well as electrolytic balance; within this important system, the angiotensin- converting enzyme (ACE), present on the surface of vascular endothelial cells, in particular those of the lungs, is deputed to the con- version of angiotensin I to angiotensin II (AII), a potent vasoconstrictive peptide. The surface spike proteins of the 'severe-acute-respiratorysyndrome-coronavirus-2' (SARS-CoV-2), the etiological agent of the ongoing 'coronavirus disease 2019' (COVID-19), are able to bind the ACE2 receptors. Therefore, a hypothesis arises: could a chronic therapeutic assumption of ACE inhibitors or AII receptor antagonists have induced several elderly and middle-aged patients to be more vulnerable to the Corona virus by upregulating ACE 2 receptors [12].

Hyperbaric oxygen therapy (HBOT) is treatment designed to in- crease the oxygen level in the blood. As such, HBOT may provide a novel means of treating/ameliorating respiratory conditions associated with COVID-19. Packed red blood cell (pRBC) transfusions are a means to enhance intravascular oxygen-carrying capability, and, thus, increase the oxygenation of tissues within the body. As such, pRBC transfusions may provide another novel means of treating/ameliorating respiratory conditions associated with COVID-19. The kidney produces erythropoietin (EPO) a circulating hormone that stimulates erythropoiesis (the production of RBCs) by binding and activating the EPO receptors (EPOR) on erythroid progenitor cells. Erythropoiesis-stimulating agents given by injection were observed to significantly increase the numbers of RBCs with increased tissue perfusion and oxygenation over several weeks. The widespread availability of ESAs provides another means to be able to rapidly and efficient treat COVID-19 patients. It was suggested that that chloroquine (CQ) and hydroxy- chloroquine (HCQ) may have a therapeutic potential to raise hemoglobin levels in patients. In addition, a recent meta- analysis of clinical trials revealed that HCQ treatment among patients diagnosed with diabetes mellitus significantly reduced their levels of hemoglobin A1c. As a result, CQ and HCQ treatments may help to improve respiratory conditions related COVID-19 by a mechanism consistent with the various previously considered treatments [13].

Routine childhood immunization may protect against COVID-19. Most routine viral vaccines are either inactivated or killed vaccines stimulate $\mathrm{T}$ Helper 1 cells (CD4+) to secrete many different types of cytokines as interferon gamma, in- terleukin-2 (IL-2), and IL-12. IL-2 provokes the maturation of the killer T cell and improve the cytotoxicity of natural killer cells recognizing and destroying cells infected with viruses. Using one or combined vaccination of varicella, Hepatitis B, MMR, Poliomyelitis, or rotavirus to either protect or treat the emerging epidemic of COVID-19 [14].
Serum albumin is a multifunctional protein known to interact with a range of exogenous and endogenous compounds. It is predominately present in the extracellular space, with high concentration being reported in the skin, guts, muscles, fluids (cerebrospinal, pleural), and secretions such as sweat, tear, milk, and saliva. The well- known fact about albumin is that there is a complex relationship between the inflammation and albumin level in the extracellular matrix under various physiological and pathological conditions. Use of combinatorial drugs therapy, in which one drug inhibits the fusion and entry of the virus into the cells and another drug internalizes and targets multiple viral components inside the cell and/or cell signaling to halt the viral propagation. It is highly recommended to use albumin as a therapeutic material, stabilizer, and deliverer of the drugs (such as known antiviral drugs and traditional molecules) that could effectively target the extracellular and intracellular viral components in the therapy of patients infected with SARSCoV-2 [15]

Ritonavir, a protease inhibitor originally designed against HIV, has the highest potential in inhibiting main protease. Ritonavir was also reported effective against SARS and MERS. Based on these large-scale clinical trials with Ritonavir, in combination with other antivirals (including Lopinavir, Remdesivir, Ribavirin, Hydroxychloroquine, etc.) and adjuvants (like Ascorbic acid, Zn2+, Melatonin, etc.) can be initiated for Corona virus therapy [16]

The anti-Flu immune responses can induce bystander immunity that is expected to non-specifically augment immunity against other viral infection such as SARS-CoV-2. Furthermore, influenza vaccination itself would generate sustained immunity that overall enhance immunity against SARS-CoV-2. This would explain why the rate of SARS-CoV-2 in children is low since they catch flu more than adults do. As such, it is expected that their immune systems be often alarmed against influenza, generating bystander immunity that harness the immune responses against related viral infection. Under this setting, we hypothesize that children generate multifactorial immunity with the repeated influenza exposure that would offer bystander immune response in case they became infected with the new SARSCoV-2. It might be possible also those individuals who received prior Flu vaccination might show mild severity of COVID-19 because of Flu-induced bystander effect of the generated immune responses which itself might cross react against SARS-CoV-2 [17].

There is evidence that some patients with severe COVID-19 experience a cytokine storm with production of large quantities of interleukins, specifically IL-1 and IL-6, and tocilizumab is a potent blocking agent of the IL- 6 receptor. The evidence for tocilizumab is anecdotal, this drug does offer hope for managing patients with severe pulmonary manifestations of COVID-19. If tocilizumab does prove to be effective, it will come at a substantial cost, both economic, as it is very expensive, and therapeutic, as it can only be delivered by intravenous infusion. It is evidence that Vitamin D play a role in modulating the production of the interleukins, thus reducing the impact of influenza. There is also evidence indicating that Vitamin D supplementation can reduce excess IL- 6 levels in diabetic mice. This evidence suggest That Vitamin D deficiency might play a role in the variation in se- verity of COVID-19, such that treatment with Vitamin D would offer a simpler alternative to tocilizumab [18].

In the article, "2019-nCoV-SARS-CoV-2 (COVID-19) infection: Cruciality of Furin and relevance with cancer, 
Cigdem Usul Afsar", hypothesized that It is called as "spiky invader" because it has a role in the attachment of the virus to the host cell receptor ACE2, especially the lung alveolar epithelial cells. It is crucial to define the protease cleavage specificity of viruses that impact human or animal health. The proprotein convertase furin is found to facilitate tumour formation and progression. Furin's tissue distribution is ubiquitous and its expression has been confirmed in a large spectrum of cancers, such as lung, head and neck, colon and gynecologic cancers and sarcomas. Furin is thought to be a pro-oncogenic driver in KRAS and BRAF mutant colorectal cancer. Aberrant expression or activity of furin may result in a variety of disorders such as diabetes, cancer and coronaviruses exploit furin for the activation of their glycoproteins. Since furin is highly expressed in lungs, an enveloped virus that infects the respiratory tract may successfully exploit this convertase to activate its surface glycoprotein [19].

A model has been proposed where amantadine is able to inhibit E-channel conductance in reconstituted lipid bilayers. In a medical hypothesis of "Amantadine as a drug to mitigate the effects of COVID-19, Gonzalo Emiliano Aranda Abreu, et al.," proposed a model where amantadine could enter the channel formed by the E-protein of the coronavirus, being able to break into the hydrogen bridges formed with water, in the same way as it does with the viroporin of the influenza virus [20].

"Can pioglitazone be potentially useful therapeutically in treating patients with covid-19? Elena Carbonib et al.,", suggests that pioglitazone is able to reduce many of these inflammatory parameters and considering that the comorbidity of diabetes, hypertension and cardiovascular disorders are indicative of a general inflammation associated with metabolic syndrome and lipid profile alteration, all conditions that can be improved by pioglitazone, we are therefore suggesting that a clinical trial with this drug or with other TZD should be considered as a support therapy in COVID-19. Finally, they concluded that pioglitazone can be administered in patients that are on statins as it has been reported that the addition of pioglitazone to atorvastatin, further reduces C-reactive protein and other laboratory markers of inflammation [21].

As per the hypothesis of "Covid-19, TMPRSS2, and whether android regulation affects pandemic virus gender incidence and age distribution of disease, Steven R. Brenner", hypothesized that SARS-COV2, coronavirus spike protein undergoes proteolytic activation by Transmembrane Serine Protease 2 (TMPRSS2), to enable SARS-COV-2 to utilize the ACE2 receptor for cellular entry. TMPRSS2 is highly expressed primarily in prostate epithelium, high-grade prostate cancers and is androgen regulated. Androgen regulation may explain the paucity of cases of Covid19 in preadolescents, since they don't have the androgens to prime the TMPRSS2 cell surface protease. Covid19 has affected men, smokers and the elderly more than other groups [22].

In "Dapsone and doxycycline could be potential treatment modalities for COVID-19, Ahmed Farouka and Samar Salman", suggests that dapsone and doxycycline may be effective in blocking inflammatory storms and, therefore, be a promising treatment of severe COVID-19 patients. Dapsone, suppresses production of specific cytokine signatures as IL1 $\alpha$, IL8, IL1 $\beta$, IL6, and IL8 and tumor necrosis factor- $\alpha$. Also, doxycycline showed efficacy in COPD by inhibiting the neutrophilic inflammation and proteolytic activity that can be present in late COVID-19 [23].
"Potential effect of natural and anabolizan steroids in elderly patient with COVID-19, Fatih Karaahmeta and Ozgur Zeliha Karaahmet", suggested that endogenous pro- inflammatory modulation agent (estrogen and testosterone) may be effective in management of elderly COVID-19 by decreasing pro-inflammatory cytokine [24].

In hypothesis based on computer data, on the epigenetic dysregulation of host metabolism by SARS coronaviral infection: Potential imputations for the SARS-CoV-2 modus operandi presented by George D. Vavougios, asserts that an exploration of SARS-CoV/human transcriptomic interaction data and gene set enrichment analysis (b) a confirmatory, focused review of the literature based on the findings by (a). On the experimental level, lipid metabolism on the cellular level has been proposed as a treatment target for COVID-19; specifically, both interactions between SARS CoV's spike protein with lipid rich membrane compartments, as well as the epigenetic modulations in lipid metabolism were considered as the end-point targets for the development of small molecules, aiming to preventSARS-CoV-2infection. While the approach presented here is inherently limited due to setting its basis on the SARS-CoV proteomic interactions, it nevertheless presents in silico and literature evidence supporting SARS-CoV-2 potential to affect human metabolism. Furthermore, as genes affected by SARS-CoV infection are significantly enriched for other infections, they may represent a common interface, targeted by viruses [25].

"COVIDiagnosis-Net: Deep Bayes-SqueezeNet based diagnosis of the coronavirus disease 2019 (COVID-19) from X-ray images by Ferhat Ucara and Deniz Korkmaz", have stated a model that is working with a deep learning-based practical structure, the early stage detections of the COVID19 cases could be done to manage and control the pandemic disease. They have demonstrated an AI-based structure to outperform the existing studies. The SqueezeNet that volunteers with its light network design is adapted for the COVID-19 diagnosis with Bayesian optimization additive. Fine-tuned hyperparameters and augmented dataset made the proposed network perform much better than existing network designs and to obtain a higher COVID-19 diagnosis accuracy [26].

Mingxiang and his team suggested that convalescent plasma exhibit a neutralizing antibody response directed against the viral S protein. This antibody blocks SARS-CoV-ACE2 entry and can be detected even 24 months after infection. When there is comparison between clinical outcomes of convalescent plasma therapy verses high dose steroids pulse therapy in SARS patients with deteriorated disease it is observe that patients in the plasma group had a shorter hospital stay and lower mortality than the comparator group, and no immediate adverse effect noted after plasma infusion. As on this discussion it is concluded that convalescent plasma from recovery patient would be highly effective weapons to win the war against COVID-19 [27].

There's still a great many more to learn about SARS-CoV-2, the novel coronavirus originator of COVID-19. Besides it has been notable and satisfying to eye researchers from around the world to collaborate and split their valuable time, adroitness, and hard-merited data in the grave pursuit to control this death-dealing virus.

\section{CONCLUSION:}

Till date, there is no specific treatment that has been confirmed to the effect against COVID-19. In this period, many of the studies have published mainly on epidemiology, causes, clinical manifestations and diagnosis, prevention and control of the novel coronavirus. The above-mentioned 
measures are an ongoing research. Studies in this domain are urgently needed to minimize the impact of outbreak.

\section{CONFLICT OF INTERESTS:}

The authors declare no conflict of interests.

\section{ACKNOWLEDGEMENTS:}

The authors are thankful to Prof T. J. Sawant founder secretary Jayawant Shikshan Prasarak Mandal and Dr. V. V. Potnis Principal JSPM's Jayawantrao Sawant College of Pharmacy and Research, Handewadi, Pune for providing all necessary facilities.

\section{REFERENCES:}

1. WHO Director-General's opening remarks at the mission briefing on COVID-19. Geneva: World Health Organization; 2020. Available at

https://www.who.int/dg/speeches/detail/who-directorgeneral-s-opening-remarks-at-the-mission-briefing-on-covid19---12-march-2020. Accessed April 28, 2020.

2. Wang D, Hu B, Hu C, Zhu F, Liu X, Zhang J, Wang B, Xiang H, Cheng Z, Xiong Y, Zhao Y, Li Y, Wang X, Peng Z, “Clinical characteristics of 138 hospitalized patients with 2019 novel coronavirus-infected pneumonia in Wuhan, China" JAMA, 2020; 323(11):1061-1069.

3. Pindiprolu SKSS and Pindiprolu SH, "Plausible mechanisms of Niclosamide as an antiviral agent against COVID-19" Med Hypotheses, 2020; 140:109765.

4. Yaqinuddin A and Kashir J, "Innate immunity in COVID-19 patients mediated by NKG2A receptors, and potential treatment using Monalizumab, Cholroquine, and antiviral agents" Med Hypotheses, 2020; 140:109777.

5. Sargina G, Yavaşoğlu SI, Yavasoglu I, "Is Coronavirus Disease 2019 (COVID-19) seen less in countries more exposed to Malaria?" Med Hypotheses, 2020; 140:109756.

6. Megna M, Napolitano M, Fabbrocinia G, "May IL-17 have a role in COVID-19 infection?" Med hypothesis, 2020; 140: 109749.

7. Adamowicz J, Juszczak K, Drewa T, "May patients receiving 5alpha-reductase inhibitors be in higher risk of COVID19complications?" Med Hypotheses, 2020; 140:109751.

8. Assimakopoulos SF and Marangos M, "N-acetyl-cysteine may prevent COVID-19-associated cytokine storm and acute respiratory distress syndrome" Med Hypotheses, 2020; 140:109778.

9. Soumier A and Sirigu A, "Oxytocin as a potential defence against Covid-19?" Med Hypotheses, 2020; 140: 109785.

10. Coppolaa M and Mondolab R, "Phototherapeutics and SARSCoV-2 infection: Potential role of bioflavonoids" Med Hypotheses, 2020; 140: 109766.

11. Bahbah EI, Negida A, Nabet MS, "Purposing Saikosaponins for the treatment of COVID-19" Med Hypotheses, 2020; 140:109782.
12. Roncati L, Gallo G, Manenti A, Palmieri B, "Renin-angiotensin system: The unexpected flaw inside the human immune system revealed by SARS-CoV-2" Med hypothesis, 2020; 140: 109686.

13. Geier MR and Geier DA, "Respiratory conditions in coronavirus disease 2019 (COVID-19): Important considerations regarding novel treatment strategies to reduce mortality" Med hypothesis, 2020; 140:109760.

14. Salmana S and Salemb ML, "Routine childhood immunization may protect against COVID-19" Med hypotheses, 2020; 140:109689.

15. Mishra PM, Uversky VN, Nandi CK, "Serum albumin-mediated strategy for the effective targeting of SARS-CoV-2" Mel Hypothesis, 2020; 140: 109790.

16. Choudhurya S and Mazumderb MK, "Suggesting Ritonavir against COVID-19/SARS-CoV-2" Med hypothesis, 2020; 140:109764.

17. Salem ML and El-Hennawy D, "The possible beneficial adjuvant effect of influenza vaccine to minimize the severity of COVID-19" Med hypothesis, 2020; 140:109752.

18. Silberstein M, "Vitamin D: A simpler alternative to tocilizumab for trial in COVID-19?" Med Hypotheses, 2020; 140:109767.

19. Afsar CU, "2019-nCoV-SARS-CoV-2 (COVID-19) infection: Cruciality of Furın and relevance with cancer" Med Hypotheses, 2020; 140:109770.

20. Abreu GEA, Aguilar MEH, Covarrubias DH, Durán FR, "Amantadine as a drug to mitigate the effects of COVID-19" Med Hypotheses, 2020; 140: 109755.

21. Carboni E, Carta AR, Carboni E, “Can pioglitazone be potentially useful therapeutically in treating patients with covid-19?" Med Hypotheses, 2020; 140:109776.

22. Brenner SR, "Covid-19, TMPRSS2, and whether android regulation affects pandemic virus gender incidence and age distribution of disease" Med Hypotheses, 2020; 140:109773.

23. Farouka A and Salmanb S, "Dapsone and doxycycline could be potential treatment modalities for COVID-19" Med Hypotheses, 2020; 140:109768.

24. Karaahmeta F and Karaahmetb OZ, "Potential effect of natural and anabolizan steroids in elderly patient with COVID-19" Med Hypotheses, 2020; 140:109772.

25. Vavougios GD, "A data-driven hypothesis on the epigenetic dysregulation of host metabolism by SARS corona viral infection: Potential implications for the SARS-CoV-2 modus operandi" Med Hypotheses, 2020; 140:109759.

26. Ucara F and Korkmazb D, "COVIDiagnosis-Net: Deep BayesSqueezeNet based diagnosis of the coronavirus disease 2019 (COVID-19) from X-ray images" Med Hypotheses, 2020; 140:109761.

27. Ye M, Fu D, Ren Y, Wang F, Wang D, Zhang F, Xia X, Lv T, "Treatment with convalescent plasma for COVID-19 patients in Wuhan, China" J Med virology, 2020; https://doi.org/10.1002/jmv.25882. 